\title{
Quantitative microhaemagglutination assay for Treponema pallidum antibodies in experimental syphilis
}

\author{
ROBERT R TIGHT AND ARTHUR C WHITE \\ From the Division of Infectious Diseases, Department of Medicine, Indiana University School of \\ Medicine, Indianapolis, USA
}

SUMMARY The quantitative microhaemagglutination assay for Treponema pallidum antibodies (MHA-TP) was studied in 52 untreated and treated rabbits with experimental syphilis. Rabbits with incubating experimental syphilis were cured or inadequately treated with penicillin $G$ and some cured rabbits were later reinfected. MHA-TP conversion occurred within 45 days in untreated rabbits. Titres reached peak levels about four months after inoculation and remained relatively high for up to two years. The quantitative MHA-TP test differentiated between rabbits cured of experimental incubating syphilis and those untreated and inadequately treated. MHA-TP titres decreased after treatment given six or 12 months after inoculation but reversion did not occur. MHA-TP conversion or significant increases in titre occurred as soon as seven days after reinfection and preceded corresponding changes in a quantitative non-treponemal test. The MHATP is useful as a screening test for treponemal antibodies in rabbits. The quantitative MHA-TP in humans after treatment for syphilis and reinfection deserves further study.

\section{Introduction}

The microhaemagglutination assay for Treponema pallidum antibodies (MHA-TP) is a relatively new treponemal serological test for syphilis. Its present role was recently reviewed. ${ }^{1}$ Compared with other treponemal tests, such as the fluorescent treponemal antibody absorption (FTA-ABS) or Treponema pallidum immobilisation (TPI) tests, it has certain advantages. It uses a sensitive technique for antibody detection, passive haemagglutination. Microvolume methods require less reagent and less material.Results can be evaluated without microscopy and are easier to interpret. ${ }^{1}$ The MHA-TP test is less expensive than other treponemal tests, is relatively reliable and reproducible, ${ }^{2}$ and can be automated. ${ }^{1-4}$ Test materials are available in kit form, making the testing of numerous specimens practical. It may combine the simplicity and lower cost of non-treponemal tests with the sensitivity and specificity of treponemal tests. It is potentially useful both as a screening and as a confirmatory test for use in conjunction with non-treponemal tests. ${ }^{1}$

Address for reprints: Dr R R Tight, c/o VA Hospital, North Elm and 21 st Avenue, Fargo, North Dakota 58102, USA

Received for publication 31 December 1979
A few studies of the MHA-TP in animals with experimental treponemal infections have been reported. It appears to be more useful for screening rabbits for possible Treponema cuniculi infections than the Venereal Disease Research Laboratory (VDRL) test and can be useful in quantitating antibodies to pathogenic treponemes. ${ }^{356}$ Experience with other quantitative treponemal tests is limited, owing partly to the relative difficulty in performing them. ${ }^{7} 8$

In the present study we attempted to clarify the meaning and interpretation of the quantitative MHA-TP in rabbits with experimental $T$ pallidum infections. We determined the range and duration of MHA-TP titres (a) in untreated rabbits; (b) in rabbits treated at varying intervals after infection; and (c) in rabbits cured of experimental incubating syphilis (EIS) which were then reinfected. For comparison, a quantitative VDRL test was also performed on all specimens.

\section{Materials and methods}

EXPERIMENTAL INFECTIONS

$T$ pallidum, Nichols strain, was originally obtained from the Center for Disease Control, Atlanta, Georgia, and was maintained by serial passage in 
rabbit testes or by freezing at $-70^{\circ} \mathrm{C}$. Male New Zealand white rabbits were obtained locally. Suspensions of freshly thawed $T$ pallidum were counted by the method of Morgan and Vryonis ${ }^{9}$ and diluted appropriately, so that approximately $5 \times 10^{4}$ organisms in $0.5 \mathrm{ml}$ total volume were injected intratesticularly into one testis of each infected rabbit. During each inoculation session at least two rabbits were left untreated as controls for infectivity of the suspension. After inoculation, rabbits were examined regularly for two months or until palpable testicular induration was detected, at which time testicular material was aspirated for darkfield examination. Rabbits were considered to be infected if testicular induration developed, aspirated testicular tissue was darkfield-positive, and MHATP seroconversion occurred. Those rabbits treated within 14 days after infection which failed to develop orchitis (testicular induration and darkfield-positive testicular tissue aspirate) or a reactive MHA-TP were considered to have been infected if the untreated control animals were infected.

\section{THERAPY}

Certain rabbits were treated with single doses of benzathine penicillin $\mathrm{G}$ (BPG) or aqueous crystalline penicillin G (ACPG) 100000 units per $\mathrm{kg}$ intramuscularly. BPG doses were comparable to those used in the treatment of human syphilis ${ }^{10}$ and were presumed to cure the experimental infections because the serum penicillin activity lasted eight or more days ( $R$ Tight, unpublished observations). This is longer than the four-day duration of activity previously shown to eradicate experimental early syphilis uniformly. ${ }^{11}$ With ACPG therapy, very high serum penicillin concentrations are obtained, but they are of such short duration that single-dose therapy is "inadequate" for the uniform eradication of $T$ pallidum infections. ${ }^{12}$

\section{TREATMENT GROUPS}

Four groups of rabbits were studied. One group of 16 rabbits remained untreated. A second group received therapy during the incubating stage of infection-that is, within the first 14 days after inoculation; of these, 10 rabbits received a single dose of BPG on day 7 , and 10 on the fourteenth day, after inoculation and nine were given a single dose of ACPG on day 7. A third group of two rabbits was treated with a single dose of BPG on day 180 after inoculation. The fourth group of five rabbits was treated one year after inoculation with weekly doses of BPG for three weeks to approximate the duration of treatment of human syphilis of more than one year's duration. ${ }^{10}$

\section{REINFECTION}

Twelve rabbits cured of EIS were reinfected, 4-10 months after the initial inoculation, with $5 \times 10^{4} T$ pallidum intratesticularly in the opposite testis from that used initially. After reinfection, rabbits were examined regularly until orchitis developed.

\section{SEROLOGICAL TESTS}

Serum samples were obtained from each rabbit as follows: before infection; $7,14,30,45$, and 60 days after infection; then every two months. Postreinfection sera were obtained on days $7,14,21$, and 30. MHA-TP and VDRL slide tests were performed on all sera. The procedures recommended by the manufacturer (Sera-Tek Treponemal Antibody Test, manufactured by Fujizoki Pharmaceutical Co Ltd, Tokyo, Japan, and distributed by Ames Co, Elkhart, Indiana) and the Center for Disease Control, Atlanta, Georgia, were followed in performing the MHATP. 13

The result for any specimen with $1+$ or greater haemagglutination was interpreted as reactive. Specimens giving - or \pm results were read as nonreactive. Each specimen reactive at a 1/80 dilution was titrated by testing serial two-fold dilutions of the absorbed serum $(1 / 20)$ in absorbing diluent; the titre was read as the last serum dilution giving a reactive result. The VDRL slide test was performed as recommended by the Center for Disease Control. ${ }^{14}$

\section{Results}

\section{UNTREATED RABBITS}

All 16 untreated rabbits were followed for 12 months after infection; of these, seven were followed for a total of 24 months. Orchitis developed in all 16 untreated rabbits 15 to 50 days (mean, 24 days) after inoculation.

Serial MHA-TP and VDRL titres in untreated rabbits are shown in fig 1 . VDRL titres appeared to rise slightly faster and reach peak levels slightly earlier after inoculation than MHA-TP titres, as indicated by the slopes of the lines connecting median titres between days 14 and 60 . VDRL titres then decreased fairly rapidly even without treatment, as has been known. ${ }^{15}$ Our results confirm previous reports of both false-positive and false-negative VDRL test results in rabbits. ${ }^{15-17}$ Thus, three of 16 rabbits had reactive VDRL tests at low titres (up to $1 / 2$ ) before inoculation. Although rigorous attempts to exclude inapparent $T$ cuniculi infections were not carried out, negative preinoculation MHA-TP test results in the rabbits in this and previous studies ${ }^{17}$ suggest that apparently normal rabbits, with no evidence of treponemal infection, may show lowgrade reactivity in the VDRL test. Furthermore, one 


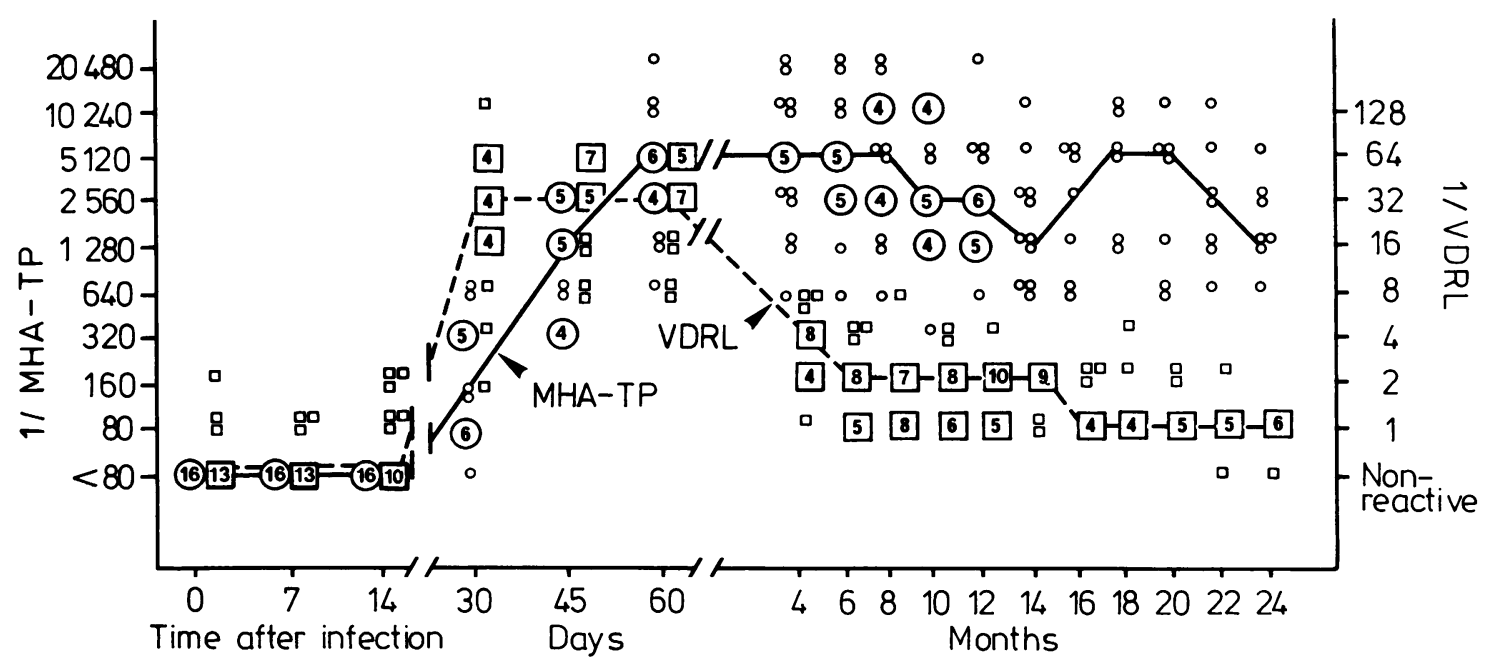

FIG 1 Range and median MHA-TP and VDRL test reciprocal titres in sera from rabbits infected with $T$ pallidum. Individual rabbit MHA-TP (O) and VDRL ( $\square$ ) reciprocal titres are shown for each time a blood sample was taken. Larger symbols indicate four or more identical results. Lines connect median reciprocal titres.

of seven rabbits had become VDRL-negative by 22 months after inoculation without treatment, confirming that treponemal tests may be necessary to detect asymptomatic treponemal infection. ${ }^{15} 16$

MHA-TP results on all sera before inoculation and on day 7 and on day 14 after inoculation were nonreactive. By day 30 however 15 of 16 sera were reactive, and by day 45 all were reactive. After conversion, MHA-TP titres remained relatively high for up to 24 months. Cox et $a l^{3}$ reported similar results. The considerable variability in titres between rabbits is illustrated in fig 1 and has been reported by others in human and experimental syphilis. ${ }^{2-4} 18$

No untreated rabbit had detectable orchitis or a reactive MHA-TP result within the initial 14 days after inoculation. Thus, EIS in this study is similar to EIS as studied by others ${ }^{19}{ }^{20}$ and to incubating human syphilis. ${ }^{21}$

RABBITS TREATED FOR EIS

Twenty rabbits with EIS were treated with BPG on day 7 or day 14 after inoculation (table). None subsequently developed orchitis. Treatment on day 7 prevented MHA-TP conversion in all rabbits. Delaying treatment until day 14 resulted in most rabbits (seven of 10) developing reactive MHA-TP results at low (compared with untreated animals) titres (table) and slightly later after inoculation. Thus, MHA-TP conversion occurred on day 30 in 15 of 16 untreated rabbits but in only three of 10 rabbits treated on day 14 ( $P<0.01$ using the "fourfold" table method of calculating $\chi^{2}$ with Yates's correction ${ }^{22}$ ). Peak MHATP titres were $\leqslant 1 / 320$ in nine of 10 rabbits treated on day 14 whereas peak titres $>1 / 1280$ occurred in all 16 untreated rabbits $(P<0 \cdot 001)$. Three rabbits treated on day 14 remained MHA-TP-negative for at least six months of observation, two reverted to non-reactive at six months, and the remainder were reactive at constantly low or, decreasing, titres $(<1 / 640)$ for up to 12 months of observation. The MHA-TP was thus useful in differentiating between rabbits cured of EIS (especially those treated on day 7) and untreated or inadequately treated rabbits.

Nine rabbits with EIS were treated on day 7 after

TABLE Peak MHA-TP titres and interval after inoculation in untreated rabbits infected with $T$ pallidum and in those treated 7 and 14 days after infection with 100000 units benzathine penicillin $\mathrm{G}$ per $\mathrm{kg}$

\begin{tabular}{|c|c|c|c|c|c|}
\hline \multirow[b]{2}{*}{ Rabbit group } & \multirow[b]{2}{*}{ No } & \multicolumn{2}{|c|}{ Reciprocal peak (MHA-TP) titre } & \multicolumn{2}{|c|}{ Time (days) after inoculation } \\
\hline & & Median & Range & Median & Range \\
\hline $\begin{array}{l}\text { Untreated } \\
\text { Treated (day 7) } \\
\text { Treated (day 14) }\end{array}$ & $\begin{array}{l}16 \\
10 \\
10\end{array}$ & $\begin{array}{l}10240 \\
<80 \\
160\end{array}$ & $\begin{array}{l}1280-20480 \\
<80 \\
<80-640\end{array}$ & $\begin{array}{l}120 \\
60^{*}\end{array}$ & $\begin{array}{l}60-360 \\
30-120^{*}\end{array}$ \\
\hline
\end{tabular}

*Values for those seven rabbits developing reactive MHA-TP results 
inoculation with the inadequate ACPG regimen. Four developed orchitis and high MHA-TP titres indicative of treatment failure. Onset of orchitis (33-50 days after inoculation; mean, 45 days) and seroconversion (on day $\mathbf{4 5}$ for all four) were slightly delayed compared with untreated rabbits. Five of the rabbits were apparently cured with the inadequate regimen. Peak titres of rabbits not cured with the ACPG regimen ( $\geqslant 1 / 5120$ at two months) were similar to peak titres in untreated animals.

\section{RABBITS TREATED SIX OR 12 MONTHS AFTER INFECTION}

Rabbits treated with BPG at six or 12 months were followed for 24 months after inoculation. MHA-TP titres of treated and untreated rabbits were similar up to the time of treatment (fig 2). After treatment MHA-TP titres decreased. The two rabbits treated at six months had 16-fold and 32-fold decreases in titre in the subsequent six months and a further fourfold decrease between 12 and 24 months. Five rabbits treated at 12 months subsequently had eight-fold to 32-fold decreases in MHA-TP titre. Such decreases were greater than the smaller fluctuations in titres that occurred in the seven untreated rabbits followed for $\mathbf{2 4}$ months. By 22 months titres in treated rabbits were significantly lower than those in the untreated animals (fig 2). Thus, although some overlap remained, six of seven rabbits treated at six or 12 months had MHA-TP titres $<1 / 320$ whereas six of seven untreated rabbits had MHA-TP titres $\geqslant 1 / 1280$ $(P<0.05)$.

\section{REINFECTION AFTER CURE OF EIS}

Results of serial post-reinfection MHA-TP and VDRL tests show some differences compared with initial infections. MHA-TP conversion occurred sooner after reinfection than after initial infection. Six of nine reinfected seronegative rabbits had reactive serum MHA-TP results seven days after reinfection whereas none of 16 specimens on day 7 was reactive after initial infections $(P<0 \cdot 01)$. Similarly, significant (fourfold and eightfold) rises in titre had occurred by day 7 after reinfection in two of three animals whose MHA-TP results before reinfection were still reactive (1/80 for all three). Relatively high MHA-TP titres also appeared to occur sooner after reinfection than after initial infection. Two rabbits had titres of $1 / 640$ by day 7 after reinfection and another three had titres $\geqslant 1 / 5120$ by day 30 after reinfection, which were higher than comparable titres after initial infections (fig 1).

After initial infections, serological conversion or significant rise in titre occurred with approximately the same rapidity (that is, usually between day 14 and day 30) with both serological tests. After reinfection, however, conversion or significant rise in titre

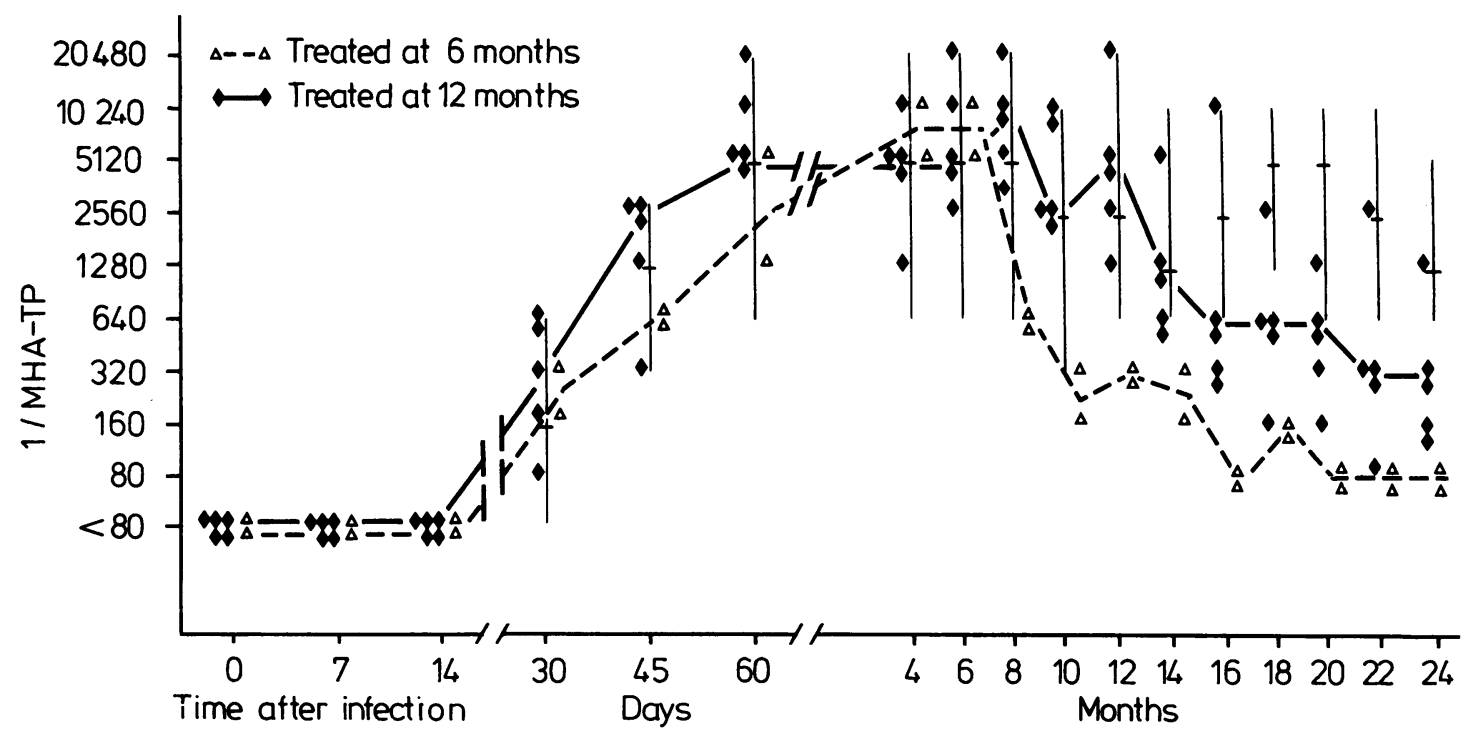

FIG 2 Range and median reciprocal MHA-TP titres in sera from two rabbits treated six months after infection and in five rabbits treated 12 months after infection. Vertical lines with short intersecting horizontal lines indicate ranges and medians of reciprocal titres in untreated rabbits (from fig 1). Other lines connect median reciprocal titres for each treatment group. 
occurred by day 7 in eight of 12 reinfected rabbits with the MHA-TP test but in none with the VDRL test $(P<0 \cdot 01)$. Overall, MHA-TP conversion or significant rise in titre preceded corresponding VDRL changes in nine of 12 rabbits and occurred simultaneously on day 30 in the other three. Eight reinfected rabbits developed orchitis 19 to 32 days (mean, 25 days) after reinoculation. The other four were killed between day 14 and 40 after reinfection, possibly before orchitis could develop.

\section{Discussion}

Results confirmed that the MHA-TP is useful for screening rabbits to be used for studies of experimental syphilis. ${ }^{36}$ The VDRL slide test may give reactive results in up to $40 \%$ of apparently normal rabbits. ${ }^{17}$ False-positive MHA-TP results in rabbits not infected with pathogenic treponemes have not, to our knowledge, been reported, although three chimpanzees experimentally infected with $T$ pallidum had pre-existing MHA-TP titres of $1 / 320$ to $1 / 5120$, and reactive FTA-ABS test results of unknown origin. ${ }^{3}$ Conversely, nine of nine rabbits infected with $T$ pertenue, 10 of 10 infected with $T$ cuniculi, and 54 of 55 infected with $T$ pallidum had reactive MHA-TP results. ${ }^{3}$ The specimen giving the one nonreactive result may have been taken on day 20 after inoculation, before MHA-TP conversion occurred.

The FTA-ABS test has been the most satisfactory serological test in experimental $T$ pallidum infections in rabbits, with a specificity approaching $100 \%$, greater sensitivity than the TPI test, and greater sensitivity and specificity than the VDRL slide test. ${ }^{15} 16$ However, performance of the FTA-ABS test on rabbit sera requires the use of fluorescein-labelled antirabbit globulin. In one study, two to three absorptions of the globulin with liver or bone marrow powder were sometimes necessary to remove non-specific staining. ${ }^{16}$ Thus, the MHA-TP test on rabbit sera seems simpler to perform than the FTAABS test. Comparative studies of the two tests in experimental syphilis would be useful. If the specificity and sensitivity of the two tests were similar, the technical simplicity of the MHA-TP test could make it preferable for screening rabbits for pre-existing treponemal antibodies in studies of experimental syphilis.

In this study, the quantitative MHA-TP test was useful in differentiating curative treatment of EIS from untreated or inadequately treated EIS. Treatment of EIS on day 7 completely prevented orchitis and seroconversion. In contrast, untreated rabbits, and rabbits not cured with the inadequate ACPG regimen, developed orchitis and high MHA-TP titres. Results of this study suggest that rabbits with
EIS could be treated on day 7 with drug regimens of similar duration to those used in the treatment of gonorrhoea in humans. Effective regimens would uniformly prevent orchitis and seroconversion. Similar animal studies ${ }^{19}$ led to human studies ${ }^{21}$ that established the effectiveness of the current aqueous procaine penicillin G (APPG) (4 800000 units) treatment regimen for gonorrhoea in eradicating human incubating syphilis. In contrast, spectinomycin failed to eradicate EIS, ${ }^{20}$ which suggests it would also be ineffective in humans. If tetracycline, ampicillin, and amoxycillin were effective in such studies, the results would support the recent suggestion that patients with incubating syphilis are "likely to be cured" by recommended schedules for the treatment of gonorrhoea with these drugs. ${ }^{23}$ Newer drugs for gonorrhoea, such as cefoxitin and cefuroxime, could be similarly studied. Groups of animals large enough to detect regimens which are inadequate in curing all infections, yet capable of eradicating some, would be needed. The ACPG regimen in this study was an example of such an inadequate regimen, and similar ACPG therapy failed to cure a case of incubating human syphilis. ${ }^{12}$ Similarly, APPG (600 000 units), tetracycline (3 g), and chloramphenicol regimens eradicated incubating syphilis in some, but not all, patients treated. ${ }^{21}$

Although the MHA-TP did not become nonreactive when treatment was first given six or 12 months after infection (like the FTA-ABS test after treatment of human syphilis), treatment was followed by significantly decreasing titres. Results of reinfection of rabbits cured of EIS suggested that the quantitative MHA-TP was an earlier indicator of reinfection than the quantitative VDRL slide test. We are unaware of any other studies of the quantitative MHA-TP after therapy or reinfection in experimental syphilis, although Collart et $a l^{7}$ showed that the quantitative TPI test titre fell more rapidly after treatment two years after inoculation than in untreated controls. Whether or not similar results occur in humans is unclear. Logan and Cox reported significant decreases in MHA-TP titres in 11 of 21 patients with early syphilis one year after therapy, ${ }^{4}$ whereas O'Neill reported slowly decreasing MHATP titres after treatment. ${ }^{24}$

In general, important shortcomings of studies of the quantitative MHA-TP in humans have been the small numbers of patients and relatively short follow up. ${ }^{12424}$ Similarly, it is not known if the MHA-TP would be a more sensitive indicator of reinfection than a non-treponemal test in humans, although in one case it was. ${ }^{25}$ Such data from human studies would clearly be of interest and could readily be obtained by performing sequential quantitative nontreponemal and MHA-TP tests on follow-up sera 
from patients treated for syphilis. Even if MHA-TP titres decreased in humans after effective treatment, this might not be of any practical importance, since quantitative non-treponemal tests are satisfactory for post-treatment follow up. However, the possibility that the quantitative MHA-TP could be an earlier indicator of reinfection has potential clinical importance, since it might enable earlier diagnosis, prompter therapy, and interruption of spread of infectious syphilis. Thus, further studies of the quantitative MHA-TP in humans after treatment for syphilis and reinfection would be worthwhile.

This work was supported in part by a grant-in-aid from the Attorney General of Indiana Public Health Trust. We thank Phillip Sylbert (deceased), Ames Co, Elkhart, Indiana, for supplying kits (Sera-Tek Syphilis Serodiagnosis Kits) used for performing MHA-TP tests. We also thank James Teipen for excellent technical assistance and Dorothy Jones for typing the manuscript.

\section{References}

1. Rudolph $\mathrm{AH}$ The microhemagglutination assay for Treponema pallidum antibodies (MHA-TP), a new treponemal test for syphilis: where does it fit? J Am Vener Dis Assoc 1976; 3:3-8.

2. Coffey EM, Bradford LL, Naritomi LS, Wood RM. Evaluation of the qualitative and automated quantitative microhemagglutination assay for antibodies to Treponema pallidum. Appl Microbiol 1972; 24:26-30.

3. Cox PM, Logan LC, Norins LC. Automated, quantitative microhemagglutination assay for Treponema pallidum antibodies. Appl Microbiol 1969; 18:485-9.

4. Logan LC, Cox PM. Evaluation of a quantitative automated microhemagglutination assay for antibodies to Treponema pallidum. Am J Clin Pathol 1970;53:163-6.

5. Okamoto S, Yoshitsugu T. Studies on Treponema pallidum haemagglutination antibodies. I TPHA antibodies in experimental syphilitic rabbits. Br J Vener Dis 1971;47:77-80.
6. Tight RR, Perkins RL. Treponema pallidum infection in subcutaneous polyethylene chambers in rabbits. Infect Immun 1976; 13: 1606-12.

7. Collart P, Franceschini P, Durel P. Experimental rabbit syphilis. Br J Vener Dis 1971;47:389-400.

8. Turner TB, Hollander DH. Biology of the treponematoses. Monograph Series No 35, Geneva: World Health Organisation, 1957: 158-66.

9. Morgan HJ, Vryonis GP. A method for the quantitation of inocula in experimental syphilis. Am J Syph Gonorrhea Vener Dis 1938; 22:462-9.

10. US Department of Health, Education, and Welfare. Syphilis, CDC recommended treatment schedules. Morbidity Mortality Weekly Rep 1976; 25: 101-7.

11. Arnold RC, Mahoney JF, Cutler JC. Reinfection in experimental syphilis in rabbits following penicillin therapy. II Reinfection in early latent syphilis. Am J Syph Gonorrhea Vener Dis 1947; 31:489-92.

12. Wright JT. Single-dose penicillin therapy. $\mathrm{Br} J$ Vener Dis 1975;51:410.

13. US Department of Health, Education and Welfare. Microhemagglutination assay for Treponema pallidum antibodies (MHA-TP); provisional technique. Atlanta GA: Center for Disease Control, 1977.

14. US Department of Health, Education and Welfare. Manual of Tests for Syphilis. Washington DC: US Government Printing Office, 1969: 33-9.

15. Miller JM. Spirochetes in Body Fluids and Tissues. Springfield, Illinois: Charles C Thomas, 1971:3-58.

16. Mothershed SM, Yobs AR, Clark JW. Comparison of the VDRL slide, TPI, and FTA-ABS tests in experimental syphilis in rabbits. Br J Vener Dis 1967; 43:267-71.

17. Pannu JS, Rosenberg MA, Israel CW, Smith JL. Incidence of reactive VDRL tests in the normal rabbit. $\mathrm{Br} J$ Vener Dis 1967; 43: 114-6.

18. O'Neill P, Warner W, Nicol CS. Treponema pallidum haemagglutination assay in the routine serodiagnosis of treponemal disease. Br J Vener Dis 1973; 49:427-31.

19. Lucas JB. The effects of gonorrhea therapy on incubating syphilis. In: Nicholas L, ed. Sexually Transmitted Diseases. Springfield, Illinois: Charles C Thomas, 1973:71-83.

20. Petzoldt D. Effect of spectinomycin on $T$ pallidum in incubating experimental syphilis. $B r J$ Vener Dis 1975;51:305-6.

21. Schroeter AL, Turner RH, Lucas JB, Brown WJ. Therapy for incubating syphilis, effectiveness of gonorrhea treatment. JAMA 1971;218:711-3.

22. Hill AB. Principles of Medical Statistics. 9th ed. New York: Oxford University Press, 1971:172-4.

23. US Department of Health, Education, and Welfare. Gonorrhea, CDC recommended treatment schedules. Morbidity Mortality Weekly Rep 1979; 28: 13-21

24. O'Neill P. A new look at the serology of treponemal disease. Br J Vener Dis 1976; 52:296-9.

25. Jephcott AE, Beveridge MAM, Sequeira PJL. Early TPHA response in primary syphilis. Br J Vener Dis 1977;53:147. 